\title{
Imaging Recommendations for Acute Stroke and Transient Ischemic Attack Patients: A Joint Statement by the American Society of Neuroradiology, the American College of Radiology, and the Society of Neurolnterventional Surgery
}

\author{
M. Wintermark, P.C. Sanelli, G.W. Albers, J. Bello, C. Derdeyn, S.W. Hetts, M.H. Johnson, C. Kidwell, M.H. Lev, D.S. Liebeskind,
} H. Rowley, P.W. Schaefer, J.L. Sunshine, G. Zaharchuk, and C.C. Meltzer

\begin{abstract}
SUMMARY: Stroke is a leading cause of death and disability worldwide. Imaging plays a critical role in evaluating patients suspected of acute stroke and transient ischemic attack, especially before initiating treatment. Over the past few decades, major advances have occurred in stroke imaging and treatment, including Food and Drug Administration approval of recanalization therapies for the treatment of acute ischemic stroke. A wide variety of imaging techniques has become available to assess vascular lesions and brain tissue status in acute stroke patients. However, the practical challenge for physicians is to understand the multiple facets of these imaging techniques, including which imaging techniques to implement and how to optimally use them, given available resources at their local institution. Important considerations include constraints of time, cost, access to imaging modalities, preferences of treating physicians, availability of expertise, and availability of endovascular therapy. The choice of which imaging techniques to employ is impacted by both the time urgency for evaluation of patients and the complexity of the literature on acute stroke imaging. Ideally, imaging algorithms should incorporate techniques that provide optimal benefit for improved patient outcomes without delaying treatment.
\end{abstract}

S troke is a leading cause of death and disability worldwide. Imaging plays a critical role in evaluating patients suspected of acute stroke and transient ischemic attack (TIA), especially before initiating treatment. Over the past few decades, major advances have occurred in stroke imaging and treatment, including Food and Drug Administration approval of recanalization therapies for treatment of acute ischemic stroke. The primary goal of imaging patients with acute stroke symptoms is to distinguish between hemorrhagic and ischemic stroke. In ischemic stroke patients,

From the Departments of Radiology, Neurology, Neurosurgery, and Biomedical Engineering, University of Virginia, Charlottesville, Virginia (M.W.); Department of Radiology, Weill Cornell Medical College, NewYork-Presbyterian Hospital, New York, New York (P.C.S.); Stanford Stroke Center, Stanford University, Stanford, California (G.W.A.); Department of Radiology and Neurosurgery, Montefiore Medical Center, Albert Einstein College of Medicine, Bronx, New York (J.B.); Mallinckrodt Institute of Radiology, Stroke and Cerebrovascular Center, Washington University School of Medicine, St. Louis, Missouri (C.D.); Departments of Radiology and Biomedical Imaging, San Francisco General Hospital and San Francisco VA Medical Center, University of California, San Francisco, San Francisco, California (S.W.H.); Departments of Diagnostic Radiology, Neurosurgery and Interventional Neuroradiology, Yale University School of Medicine, New Haven, Connecticut (M.H.J.); Departments of Neurology and Georgetown University Stroke Center, Georgetown University Medical Center, Washington, DC (C.K.); Department of Radiology, Harvard Medical School, Massachusetts General Hospital, Boston, Massachusetts (M.H.L., P.W.S.); Department of Neurology, Stroke Center, University of California, Los Angeles, Los Angeles, California (D.S.L.); Departments of Radiology, Neurology, and Neurosurgery, University of Wisconsin School of Medicine and Public Health, Madison Wisconsin (H.R.); Departments of Radiology, Neurology, and Neurosurgery, Case Western Reserve University, Cleveland, Ohio (J.L.S.); Department of Radiology, Stanford University and Stanford University Medical Center, Stanford, California (G.Z.); and Departments of Radiology and Imaging Sciences, Neurology, and Psychiatry and Behavioral Sciences, Emory University School of Medicine, Atlanta, Georgia (C.C.M.). secondary goals of imaging before initiating revascularization interventions with intravenous thrombolysis or endovascular therapies include identification of the location and extent of intravascular clot as well as the presence and extent of "ischemic core" (irreversibly damaged tissue) and "penumbra" (hypoperfused tissue at risk for infarction). ${ }^{1-3}$ In addition, early identification of the stroke etiology or mechanism (eg, carotid atherosclerotic disease, vascular dissection, or other treatable structural causes) is critical to treatment decisions and long-term management.

A wide variety of imaging techniques has become available to assess vascular lesions and brain tissue status in acute stroke patients. However, the practical challenge for physicians is to understand the multiple facets of these imaging techniques, including which imaging techniques to implement and how to optimally use them, given available resources at their local institution. Important considerations include constraints of time, cost, access to imaging modalities, preferences of treating physicians, availability of expertise, and availability of endovascular therapy. The choice of which imaging techniques to

M. Wintermark and P.C. Sanelli are co-first authors of this article.

A shortened version of this article is published in the Journal of the American College of Radiology. ${ }^{4}$

Please address correspondence to Max Wintermark, MD, MAS, Department of Radiology, Neuroradiology Division, University of Virginia, Box 800170, Charlottesville, VA 22908; e-mail: Max.Wintermark@gmail.com

- Indicates open access to non-subscribers at www.ajnr.org

http://dx.doi.org/10.3174/ajnr.A3690 


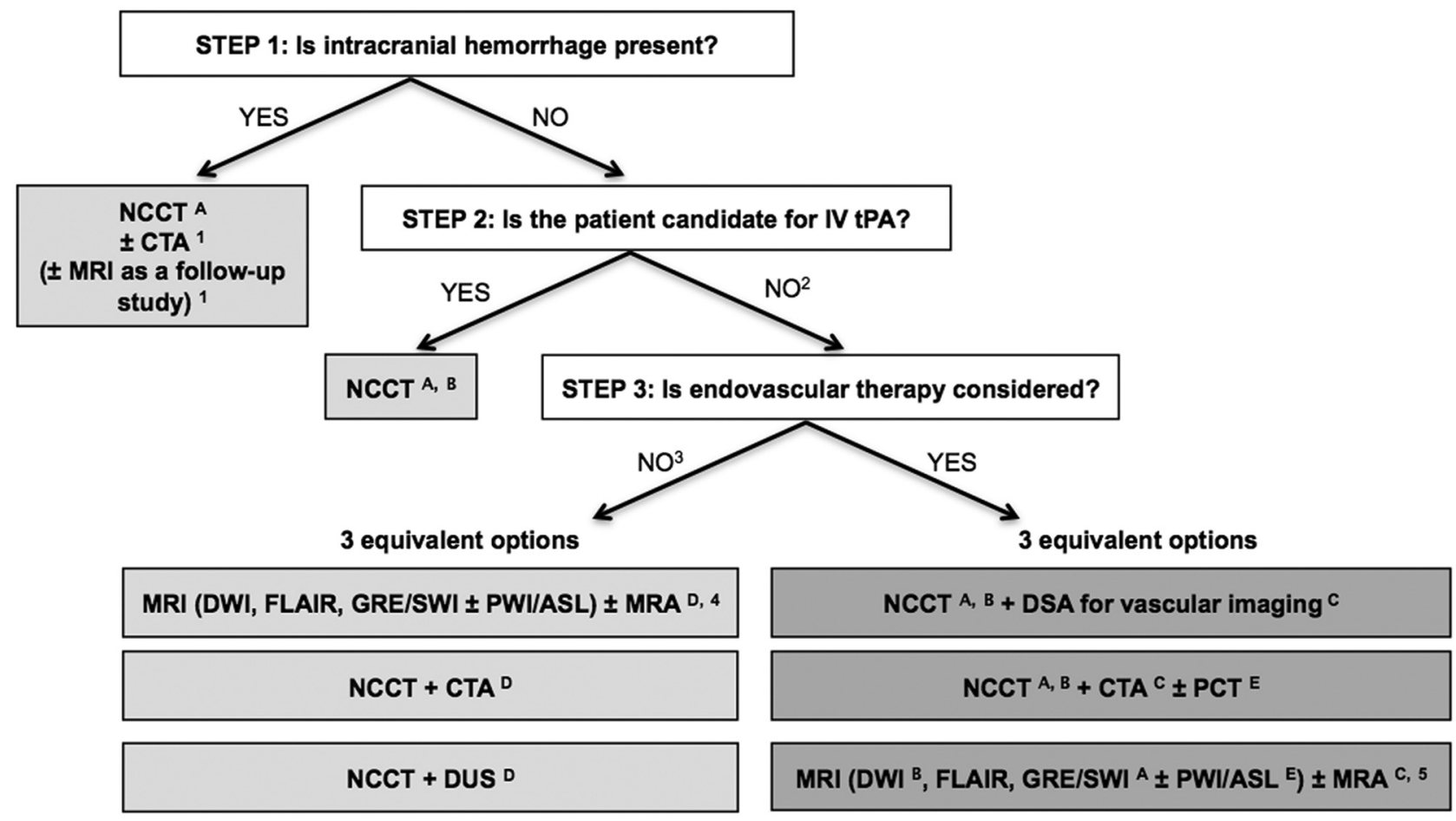

FIG 1. Suggested imaging protocols for patients presenting with acute stroke symptoms based on the clinical scenario and the therapeutic options considered and available. Each of the gray boxes represents 1 imaging strategy. In order not to delay treatment, a standardized imaging approach should be used: One imaging strategy (gray box) should be selected, and all imaging studies belonging to this strategy should be performed upfront in as few sessions as possible:

1) To assess the etiology of the intracranial hemorrhage (CTA for vascular pathologies, such as aneurysms, arteriovenous malformations, vasculopathies; MR imaging for vascular malformations, neoplastic and other pathologies associated with hemorrhage).

2) Also if the patient is not a candidate for IV TPA (contraindication to IPA, outside the time window for tPA) or if IV tPA failed or it is thought that it may fail.

3) For patients who are outside the time window for acute reperfusion therapies ( $>4.5$ hours at sites where only IV tPA is being considered; $>8$ hours at sites where endovascular therapy is considered) and for patients with TIAs, emphasis is on secondary prevention and their imaging work-up should be focused on vascular imaging (CTA, MRA or Doppler-ultrasound [DUS]) to assess carotid arteries as a possible cause of the ischemic stroke, with secondary prevention in mind. If MRA is obtained, it makes sense to concurrently obtain MR imaging with DWI, FLAIR, and GRE/SWI. Echocardiography should also be obtained to assess for cardiac sources.

4) If available, MR imaging/MRA is the preferred imaging technique for TIA patients.

5) At institutions where MR imaging is available $24 / 7$ and can be performed within a short time after admission.

A) To assess for intracranial hemorrhage.

B) To assess the extent of ischemic core.

C) To assess the location and extent of the intravascular clot.

D) To assess carotid atherosclerotic disease.

E) To assess the extent of viable tissue.

employ is impacted by both the time urgency for evaluation of patients and the complexity of the literature on acute stroke imaging. Ideally, imaging algorithms should incorporate techniques that provide optimal benefit for improved patient outcomes without delaying treatment. Therefore, it is most practical and efficient to use a standardized imaging approach, with all relevant imaging studies conducted in as few sessions as possible (Fig 1).

We performed a review of the evidence in the literature on the utility of various imaging techniques in acute stroke and TIA patients to establish best practices with standardization of imaging protocols. We indicated the quality of publications for diagnostic tests and interventions by assigning levels of evidence (Tables 1 and 2). These levels of evidence are based on the Oxford Centre for Evidence-based Medicine-Levels of Evidence. ${ }^{3 a}$ The goal of this article is to present practical imaging recommendations for patients presenting with acute stroke and TIA across different practice settings and to provide the rationale and evidence sup- porting their use. These recommendations are in agreement with the American College of Radiology Appropriateness Criteria. ${ }^{4} \mathrm{We}$ recognize that stroke imaging is a rapidly evolving field and that a number of the recommendations presented are the topic of continued investigation.

\section{Rationale and Imaging Evidence for Patients Presenting with Acute Stroke Symptoms}

The initial step in the evaluation of patients with symptoms of acute stroke is to differentiate between hemorrhagic and ischemic stroke (Fig 1). For patients with acute ischemic stroke who are candidates for IV tissue plasminogen activator, a noncontrast CT of the head should be obtained to determine eligibility for treatment. IV tPA can then usually be initiated without waiting for further imaging. In patients under consideration for endovascular therapy, 3 imaging options may be used: 1) NCCT followed immediately by digital subtraction angiography for vascular assessment, 2) NCCT + CT angiography \pm perfusion CT (PCT), or 3) 
MR imaging $( \pm$ PWI/ASL $)+$ MR angiography at institutions that can offer MR imaging 24/7 without delaying treatment. In patients who are not candidates for IV or endovascular therapy and in patients with TIA, vascular imaging is recommended to guide management for secondary prevention of future stroke.

\section{Imaging Evidence for Assessing Intracranial Hemorrhage}

NCCT is the accepted standard-of-care imaging technique for exclusion of intracranial hemorrhage and has been incorporated in the inclusion criteria in randomized clinical trials evaluating the efficacy of IV thrombolysis. ${ }^{4,5}$ NCCT is often referred to as the "reference standard" for detection of acute intracranial hemorrhage based on reports describing its accuracy with early CT scanners. ${ }^{6,7}$ However, there are no recent studies that have used a true reference standard, such as surgical or pathologic confirmation, to support level I evidence. Therefore, the sensitivity and specificity of NCCT in detecting intracranial hemorrhage are unknown. The many advantages of NCCT in the emergent setting, as well as

\begin{tabular}{|c|c|}
\hline $\begin{array}{l}\text { Levels of } \\
\text { Evidence }\end{array}$ & Type of Evidence \\
\hline la & $\begin{array}{l}\text { Systematic review (with homogeneity) }{ }^{b} \text { of level } 1 \\
\text { studies }^{c}\end{array}$ \\
\hline $\mathrm{lb}$ & Level 1 studies ${ }^{c}$ \\
\hline \multirow[t]{2}{*}{ II } & Level 2 studies ${ }^{d}$ \\
\hline & Systematic reviews of level 2 studies \\
\hline \multirow[t]{2}{*}{ III } & Level 3 studies $^{\mathrm{e}}$ \\
\hline & Systematic reviews of level 3 studies \\
\hline IV & $\begin{array}{l}\text { Consensus, expert committee reports or opinions } \\
\text { and/or clinical experience without explicit } \\
\text { critical appraisal; or based on physiology, bench } \\
\text { research, or "first principles" }\end{array}$ \\
\hline
\end{tabular}

adapted from Reference 3a.

b "Homogeneity" means there are no or minor variations in the directions and degrees of results between individual studies that are included in the systematic review. 'Level 1 studies are studies:

- that use a blind comparison of the test with a validated reference standard

- in a sample of patients that reflects the population to whom the test would apply.

${ }^{d}$ Level 2 studies are studies that have only 1 of the following:

- narrow population (the sample does not reflect the population to whom the test would apply).

- use a poor reference standard (defined as that where the "test" is included in the "reference," or where the "testing" affects the "reference").

- the comparison between the test and reference standard is not blind.

- case-control studies

e Level 3 studies are studies that have at least 2 or 3 of the features listed above. the proved benefit of IV thrombolysis in patients selected by NCCT,${ }^{4,5}$ have led to its continued widespread use in acute stroke imaging (Table 3).

MR imaging T2*-weighted sequences have been studied for detection of acute and chronic hemorrhage in acute stroke patients. The accuracy of MR imaging techniques for detection of intracranial hemorrhage in the acute stroke setting (within 6 hours) has been reported as likely equivalent to NCCT (level Ib). ${ }^{8,9}$ Additionally, T2*-weighted sequences (including gradient-recalled echo [GRE] and susceptibility-weighted imaging sequences) have superior accuracy in the detection of chronic microhemorrhages. ${ }^{9-11}$ In 1 large study as well as a meta-analysis, there was no statistically significant increased risk of symptomatic hemorrhage when patients with a small number of chronic microhemorrhages $(<5)$ were treated with IV thrombolysis (level Ia). ${ }^{12,13}$ However, the risk of symptomatic hemorrhage in patients with numerous chronic microhemorrhages undergoing treatment with IV thrombolysis is unknown. It is important to recognize that the pivotal CT-based trials proving a benefit for IV tPA likely included patients with multiple microhemorrhages.

If intraparenchymal hemorrhage is present, as in $15 \%$ of all strokes, the imaging evaluation in the acute phase may include CTA of the intracranial arteries for evaluation of an underlying vascular malformation. ${ }^{14-16}$ CTA may demonstrate a "spot sign,"

Table 3: Advantages and limitations of CT and MR imaging

\begin{tabular}{lcc}
\multicolumn{1}{c}{ Imaging Characteristics } & CT MRI \\
\hline Availability in the acute setting (0-6 hours) & ++ & - \\
Rapid image acquisition & ++ & + \\
Lack of vulnerability to motion artifacts & + & - \\
Accessibility for patients with monitors & ++ & - \\
$\quad$ and/or ventilators & & \\
Feasibility and safety for patients with metallic implants & ++ & - \\
$\quad$ (pacemakers, implantable defibrillators) & & \\
Lower cost & + & - \\
Lack of ionizing radiation & - & ++ \\
Renal toxicity associated with contrast administration & + & + \\
Time for postprocessing angiography and perfusion & - & - \\
$\quad$ imaging & & \\
Sensitivity to lacunar and posterior fossa infarcts & - & ++ \\
Differentiation between acute and chronic ischemia & - & ++ \\
Ability to assess causes of ICH or SAH while in the & + & + \\
$\quad$ scanner & & \\
Detection of chronic hemorrhage including microbleeds & - & + \\
\hline
\end{tabular}

Note:-ICH indicates intracranial hemorrhage.

Table 2: Levels of evidence for intervention studies ${ }^{a}$

\begin{tabular}{cl}
\hline Levels of Evidence & Type of Evidence \\
\hline la & High-quality meta-analyses, systematic reviews of RCTs, or RCTs with a very low risk of bias \\
$\mathrm{lb}$ & Well-conducted meta-analyses, systematic reviews of RCTs, or RCTs with a low risk of bias \\
$\mathrm{lc}$ & Meta-analyses, systematic reviews of RCTs, or RCTs with a high risk of bias ${ }^{\mathrm{b}}$ \\
$\mathrm{2a}$ & High-quality systematic reviews of case-control or cohort studies; high-quality case-control or cohort studies \\
& with a very low risk of confounding, bias, or chance and a high probability that the relationship is causal \\
$2 \mathrm{~b}$ & Well-conducted case-control or cohort studies with a low risk of confounding, bias, or chance and a moderate \\
& probability that the relationship is causal \\
& Case-control or cohort studies with a high risk of confounding bias or chance and a significant risk that the \\
& relationship is not causal \\
& Nonanalytic studies (for example, case reports, case series) \\
5 & Expert opinion, formal consensus \\
\hline
\end{tabular}

Note:-RCT indicates randomized controlled trial.

${ }^{\text {a }}$ Adapted from Reference $3 \mathrm{a}$.

${ }^{\mathrm{b}}$ Studies with a level of evidence indicated by_ should not be used as a basis for making a recommendation. 
indicative of active bleeding, predictive of hematoma expansion, and strongly associated with poor outcomes. ${ }^{17-20}$ An MR imaging without and with contrast is sometimes obtained to assess for an underlying neoplastic or vascular mass, or associated microhemorrhages that may suggest amyloid angiopathy, multiple cavernous malformations, or septic emboli among other etiologies. In the acute phase, sensitivity of MR imaging may be limited by mass effect from the hematoma and the complex MR imaging signal of blood products that may obscure subtle enhancing lesions; its sensitivity is improved in the subacute phase once the hematoma has been resorbed. ${ }^{14-16}$ Please note that the imaging evaluation of patients with aneurysmal subarachnoid hemorrhage is beyond the scope of this article.

\section{Rationale and Imaging Evidence for Acute Ischemic Stroke Patients Who Are Candidates for IV Thrombolysis}

Treatment options are considered for patients with acute ischemic stroke without intracranial hemorrhage present on imaging. FDA guidelines for administration of IV thrombolysis include imaging to exclude intracranial hemorrhage and its interpretation by a physician with appropriate expertise, while the completion of this initial imaging within 45 minutes of the patient admission to the emergency department is a Centers for Medicare and Medicaid Services Quality Reporting measure. ${ }^{21-23}$ There is strong evidence supporting the use of IV tPA as a recanalization therapy to improve clinical outcomes during the 0 - to 3 -hour time window (level 1a) ${ }^{24-26}$ and during the 3- to 4.5-hour time window (level 1b). ${ }^{27-29}$ This benefit is despite an increased risk of symptomatic intracranial hemorrhage after infusion. Overall, there is strong evidence (level Ia) supporting the timely use of imaging of the brain to exclude hemorrhage in patients with the clinical diagnosis of stroke and before initiating IV thrombolytic therapy. ${ }^{4,24}$ The primary goals of imaging during the 0 - to 4.5 -hour time window are to exclude the presence of intracranial hemorrhage and assess the presence and extent of ischemic changes. The presence of intracranial hemorrhage (excluding microbleeds) is an absolute contraindication to administering IV thrombolytic therapy. Early signs of ischemia involving more than one-third of the middle cerebral artery territory in the 0 - to 6-hour time window have been associated with large infarcted regions, increased risk of hemorrhagic transformation, and poor outcomes and thus constitute a relative contraindication to IV thrombolysis. ${ }^{26,30,31}$

Imaging in patients who are potential candidates for IV thrombolysis should not delay administration of IV thrombolysis, as "time is brain." 22 Therefore, IV tPA decisions should be made immediately after the NCCT is completed. At institutions that offer endovascular treatment to IV tPA-eligible patients with large-artery occlusion (likely tPA failures), additional imaging can be performed while IV tPA is prepared/administered, to not delay treatment. From a logistics perspective, institutions should develop a standardized imaging algorithm based on their capabilities and interpretation of current evidence. This imaging protocol should be adhered to for all eligible patients to expedite the process and minimize delays in treatment. For instance, if NCCT, CTA, and MR imaging constitute the imaging algorithm selected by an institution to evaluate for potential endovascular candidates, NCCT and CTA should be obtained in 1 imaging session to minimize imaging time. At institutions performing it regularly, the entire multimodal CT evaluation does not delay patient care. Specifically, it does not delay IV thrombolysis, which can be performed directly in the CT scanner once the NCCT is completed and while the CTA and/or PCT are being obtained (level 2b). ${ }^{32-35}$ Few institutions are able to perform MR imaging studies in the acute setting. Such MR imaging studies are usually performed after the NCCT has been completed (or used as a replacement for it) and are often obtained during or following IV tPA administration.

\section{Imaging Evidence for Detection of Ischemia}

NCCT is also used to assess for early signs of infarction, including loss of gray-white differentiation, sulcal effacement, and hyperattenuated clot in the proximal vessels. ${ }^{30,36}$ NCCT has been reported to have low sensitivity (39\%) and high specificity (100\%) for detection of ischemic changes (level Ia). ${ }^{30,37}$ However, the significance of these early signs detected on NCCT has been debated. In the European Cooperative Acute Stroke Studies (ECASS), large infarctions with early swelling had an increased incidence of hemorrhage and poor outcome following thrombolytic therapy. ${ }^{30,37}$ Conversely, the National Institute of Neurologic Disorders and Stroke rtPA Stroke Trial reported that extensive early signs of infarction on NCCT were associated with stroke severity but not with adverse outcome after thrombolysis. ${ }^{36}$ However, more recent studies have disagreed and recommended criteria for withholding IV thrombolytic therapy in the 0 - to 3-hour time window for definite signs of ischemia involving more than one-third of the MCA territory. ${ }^{38}$

Detection of early signs of ischemia on NCCT varies among experienced observers, ${ }^{39-41}$ depending on the size of the infarction, the time between symptom onset and imaging, and the CT window and level settings used. A more objective approach to define the extent of early ischemic changes has been described in the Alberta Stroke Program Early CT Score (ASPECTS), which is a 10-point scoring system of the MCA territory. ${ }^{42-44}$ Although ASPECTS showed superior interobserver agreement, it only modestly improved accuracy for predicting functional outcome and performed the same as the one-third MCA rule for predicting symptomatic hemorrhage. ${ }^{42}$ Specifically, an ASPECTS score $\leq 7$ has been shown to predict poor functional outcome with $78 \%$ sensitivity and $96 \%$ specificity and symptomatic hemorrhage with $90 \%$ sensitivity and $62 \%$ specificity. ${ }^{23}$

Of note, the source images from CTA (CTA-SI) have been shown to have increased sensitivity relative to NCCT for detecting ischemic changes, except for infarcts that are small or in the posterior fossa (level II), ${ }^{45,46}$ though with current technology (rapid CT acquisition), they tend to overestimate the size of the infarct. ${ }^{47}$ Importantly, CTA-SI maps are strongly dependent on the precise timing of the imaging, which may differ between centers and between individual patients.

MR diffusion-weighted imaging is more sensitive for detecting ischemic changes compared with NCCT (level Ia). ${ }^{48-53}$ Its sensitivity in detecting ischemia is reported as $99 \%$ with a high specificity of $92 \% .{ }^{48,50,53-59}$ In anterior circulation strokes, the DWI lesion volume correlates well with baseline clinical stroke severity, final infarct volume, and clinical outcome (level II). ${ }^{60-62} \mathrm{Al}$ - 
though strong evidence suggests that MR imaging is superior to NCCT for confirming stroke within the first 24 hours (level Ia), ${ }^{53}$ logistical issues related to performing MR imaging in the emergent setting, as well as the proved benefit of CT-based selection in randomized controlled trials, limit the use of MR imaging in the emergent setting (Table 3). Therefore, MR imaging may be reserved for select patients in whom the clinical diagnosis is uncertain or for centers that have MR imaging readily available 24/7 with streamlined protocols to limit imaging time within the standard-of-care guidelines for thrombolytic therapy.

Summary. In acute stroke patients who are candidates for IV thrombolysis ( 0 - to 4.5-hour time window), NCCT or MR imaging is recommended to exclude intracranial hemorrhage and determine the extent of ischemic changes. ${ }^{4,63,64}$

1) The presence of a large acute hypoattenuation on NCCT increases the risk of hemorrhagic transformation after thrombolytic therapy. This is considered a relative, not absolute, contraindication for IV tPA. MR DWI may be obtained for a more definitive estimate of the extent of ischemia, only if this does not delay IV thrombolysis.

2) The presence of a small number of MR imaging-detected chronic microhemorrhages $(<5)$, in the absence of hemorrhage on NCCT, is not a contraindication to IV thrombolysis. However, the risk of hemorrhage in patients with multiple chronic microhemorrhages $(>5)$ is unknown.

\section{Rationale and Imaging Evidence for Acute Ischemic Stroke Patients Who Are Candidates for Endovascular Revascularization}

There is limited evidence supporting the use of intra-arterial thrombolytic agents up to 6 hours. Also, the evidence supporting improved clinical outcomes with first-generation mechanical embolectomy devices up to 8 hours following symptom onset, compared with standard medical care, has recently been challenged by the results of the Mechanical Retrieval and Recanalization of Stroke Clots Using Embolectomy (MR RESCUE), ${ }^{65}$ Interventional Management of Stroke (IMS III), ${ }^{66}$ and Intra-arterial Versus Systemic Thrombolysis for Acute Ischemic Stroke (SYNTHESIS EXP) trials. ${ }^{67}$ Mechanical thrombectomy devices received FDA approval for use in patients presenting up to 8 hours from symptom onset because of early recanalization being associated with a 4 - to 5-fold improvement in clinical outcome. ${ }^{68}$ Further randomized, controlled trials are needed to test the clinical efficacy of new-generation stent-retriever ("stentriever") thrombectomy devices.

Initiation of endovascular revascularization therapy provides targeted treatment at the site of the clot. Due to the associated risks of the procedure, if this is considered, more information for appropriate patient selection is needed to achieve an acceptable risk-benefit ratio. ${ }^{69,70}$ Poor response and poor outcomes with IV thrombolysis have been found with carotid terminus and large, proximal artery occlusions. ${ }^{71,72}$ Additionally, the outcome after endovascular therapy is also influenced by the composition and location of the thrombus, with improved recanalization rates for more proximal rather than distal thrombus. ${ }^{73-77}$ Thus, there is some justification (level II) for vascular imaging of acute stroke patients at the time of the initial brain imaging study to triage patients to the best therapy and determine prognosis. This may also be the most practical and efficient time to obtain vascular imaging in stroke patients.

There are 3 major imaging strategies (and numerous combinations of these 3 strategies) used in acute ischemic stroke patients who are considered for endovascular revascularization therapy, with different underlying rationales (Fig 1). There is currently no definitive evidence supporting one strategy over the other. Some believe that more imaging provides additional, clinically relevant information, while others are concerned about the additional time resulting from the additional imaging and the potential delay to recanalization it might cause. The choice of imaging implemented may depend on physician preference and logistical factors (such as whether advanced imaging, especially MR imaging, can be performed quickly and on a $24 / 7$ basis). In considering the underlying rationale for endovascular therapy, additional imaging may be more justified in patients within the 4.5- to 8-hour time window. In patients with a contraindication to IV tPA within the 0 - to 4.5 -hour time window and in patients considered for endovascular therapy after IV tPA failure, imaging the volume of the infarct may be sufficient.

The first strategy consists of going to the angiography suite immediately after the initial NCCT. The rationale for this approach is to minimize the door-to-recanalization time. In this setting, the vascular patency status is assessed on the DSA that precedes the therapeutic portion of the procedure, before lysis or removal of the clot. Collateral patterns can also be demonstrated, though infarct volume can only be indirectly assessed by attention to flow, parenchymal blush, and arterial-to-venous transit times. The second strategy consists of obtaining a CTA to assess vascular patency, with or without perfusion imaging, to better characterize the site of occlusion and the ischemic tissue before making an endovascular treatment decision. The third strategy consists of using MR imaging/MRA, possibly with diffusion- and perfusionweighted imaging at institutions where it can be performed quickly and on a $24 / 7$ basis. The rationale of these latter approaches is that the extra time needed to perform this additional imaging may be justified by the information gathered and the implications for decision-making. ${ }^{78,79}$ Some studies have demonstrated that the extra time for imaging until treatment does not adversely affect outcomes. ${ }^{80-82}$

\section{Imaging Evidence for Detection of Intravascular Clot}

Vascular imaging of the acute stroke patient before endovascular therapy is necessary to determine whether an embolus/thrombus is present that is accessible and amenable to intra-arterial thrombolysis and/or mechanical thrombectomy. Imaging of the intracranial and extracranial vessels can be performed quickly and noninvasively by using CTA and MRA. However, DSA is considered the reference standard for detection of vascular stenoses and occlusions. CTA has been reported to have high sensitivity (97\%$100 \%)$ and specificity (98\%-100\%) for detecting intracranial stenoses and occlusions compared with DSA (level Ib). ${ }^{83-90}$ MRA can also be used to characterize vascular patency (level Ib). 4,64,91 CTA has been shown to be slightly superior to MRA for this purpose, typically for distal vascular lesions. ${ }^{83,84}$ Complete or partial signal void in regions of high and/or turbulent flow may occur on 
time-of-flight MRA, leading to an overestimation of stenosis. Window settings and presence of calcifications or adjacent bone can limit CTA evaluation.

CTA provides additional tissue information on the CTA-SI, initially thought to represent blood volume-weighted data. However, with current, faster CTA protocols, a steady-state is not always reached; the CTA-SI may be more blood flow-weighted and can frequently overestimate ischemic core relative to the DWI lesion volume. ${ }^{47}$ Hypoattenuated regions on CTA-SI indicate early ischemic changes that may be seen to better advantage compared with NCCT. In one study, the combined information from the CTA and CTA-SI demonstrated marked improvement in localization of both the ischemic core and the occluded vessel compared with NCCT and clinical information. ${ }^{92}$ Another advantage of CTA is that it can be obtained immediately following NCCT, after initiation of IV thrombolytic therapy in the CT scanner, to avoid delaying treatment.

\section{Imaging Evidence for Detection of Viable Tissue}

Determination of tissue viability based on imaging has the potential to individualize thrombolytic therapy and extend the therapeutic time window for some acute stroke patients. Although perfusion imaging has been incorporated into acute stroke imaging algorithms at some institutions, its clinical utility has not been proved. The potential value of perfusion imaging has been assessed in the Desmoteplase in Acute Ischemic Stroke-phase II (DIAS-II) trial by using MR diffusion/perfusion mismatch and a perfusion-CT mismatch as entry criteria to receive IV desmoteplase in patients presenting up to 9 hours from symptom onset. ${ }^{93}$ However, this trial failed to demonstrate superiority of treatment over placebo by using penumbral imaging as a selection criterion. Other trials such as Diffusion-weighted Imaging Evaluation For Understanding Stroke Evolution (DEFUSE), DEFUSE-2, and Echoplanar Imaging Thrombolysis Evaluation Trial (EPITHET) have shown promising results by using a combination of diffusion and perfusion imaging to identify good candidates for revascularization therapy beyond 3 hours. ${ }^{78,79,94}$ The MR RESCUE trial failed to demonstrate any difference in outcome in stroke patients selected by using penumbra imaging compared with no selection at all. ${ }^{65}$ Therefore, there is insufficient evidence at this point supporting the use of penumbra imaging to select patients for acute reperfusion therapy. Further randomized, controlled trials are needed to test the full spectrum of penumbra imaging selection for acute stroke therapies.

MR perfusion is employed at some institutions to assess the diffusion/perfusion mismatch. The presence of a perfusion abnormality larger than the DWI lesion (ie, a mismatch) is a qualitative marker for potential infarct expansion. ${ }^{95-97}$ However, the extent of mismatched tissue varies greatly, depending on the perfusion parameter selected and the threshold selected to represent the PWI abnormality (level 2b). ${ }^{93,98-100}$ Individual studies have reported varying perfusion parameters as most predictive of tissue viability and clinical outcome, without clear consensus. Some studies have suggested that the Tmax parameter (time-to-peak of the residue function) by using a threshold $>6$ seconds is a good predictor of infarct growth in the absence of early recanalization. ${ }^{101-103}$
Perfusion CT is another method used to assess the ischemic core and penumbra. Similar to MR PWI, there is no clear consensus on the optimal perfusion parameter that is most predictive of tissue viability and outcome. A prospective multicenter study reported that an absolute cerebral blood volume threshold reflected the ischemic core and that a relative mean-transit-time threshold most accurately reflected the penumbra. ${ }^{104}$ However, in more recent and larger studies, relative cerebral blood flow was found to be more predictive of the ischemic core (nonviable tissue) than absolute CBV. ${ }^{105-113}$ As for PWI, there is a need for standardization of the PCT methods used to define the ischemic core and the penumbra.

It is important to note that perfusion imaging has many applications beyond characterization of the penumbra and triage of patients to acute revascularization therapy. The negative results of the MR RESCUE trial do not negate these potential benefits. ${ }^{65}$ These applications include, but are not limited to, the following: 1) improving the sensitivity and accuracy of stroke diagnosis (in some cases, a lesion on PCT leads to more careful scrutiny and identification of a vascular occlusion that was not evident prospectively, particularly in the M2 and more distal MCA branches) ${ }^{46,113-115}$;2) excluding stroke mimics ${ }^{116} ; 3$ ) better assessment of the ischemic core ${ }^{114}$ and collateral flow $^{117}$; and 4) prediction of hemorrhagic transformation and malignant edema. ${ }^{118,119}$

\section{Imaging Evidence for the Characterization of Collateral Vessels}

The concept of collaterals as a vascular network that can potentially bypass devastating effects of a blocked cerebral artery has recently gained momentum. Collaterals have been shown to enhance recanalization and reperfusion, reduce the size of the core and ischemic lesion growth, decrease the risk of hemorrhagic transformation, and improve outcomes with IV and endovascular revascularization (level III). ${ }^{117,118}$ More specifically, a poor collateral pattern has a high specificity for poor tissue and clinical outcome (level III). ${ }^{120}$

Several imaging approaches have been proposed to evaluate collaterals, including CTA, PCT, perfusion-weighted imaging, DSA, arterial spin-labeling (ASL), and positron-emission tomography. Currently, none of these techniques is absolute nor is any established as a reference standard to assess and quantify collateral flow. Imaging techniques that include a serial, temporal assessment have a definitive advantage because of the dynamic nature of collateral perfusion. Optimized imaging analyses of collateral perfusion patterns may have to consider the underlying mechanism of arterial occlusion, as patterns may vary from intracranial atherosclerosis to cardioembolism. ${ }^{121}$ Thresholded volumes of hypoperfusion on perfusion maps may not be as informative as voxel-based measures that depict the heterogeneity of the penumbra. $^{122}$

Summary. In acute stroke patients who are candidates for endovascular therapy, vascular imaging (CTA, MRA, DSA) is strongly recommended during the initial imaging evaluation. ${ }^{4,57,63,64}$ Perfusion imaging may be considered to assess the target tissue "at risk" for reperfusion therapy. ${ }^{4,64}$ However, the accuracy and use- 
fulness of perfusion imaging to identify and differentiate viable tissue have not been well-established.

1) Acute large-vessel intracranial thrombus is accurately detected by CTA, MRA, and DSA.

2) Patients with large infarctions tend to have poor outcomes. The ischemic core is determined most accurately with DWI. Appropriately thresholded PCT-CBV and PCT-CBF can also be used to identify the ischemic core despite immediate reperfusion.

3) A poor collateral pattern has a high specificity for poor tissue and clinical outcome (level III).

\section{Rationale and Imaging Evidence for Acute Ischemic Stroke Patients Who Are NOT Candidates for IV or Endovascular Therapy and Patients with Transient Ischemic Attacks}

When acute revascularization therapy is not being considered, the role of imaging is primarily focused on diagnosis, prevention of immediate complications, and the identification of potentially treatable causes of future stroke. In patients with TIAs, multimodal MR imaging is preferred, and NCCT should be obtained only if MR imaging is not available, as NCCT has limited utility in patients whose symptoms have resolved. ${ }^{123}$ DWI can demonstrate lesions in approximately $40 \%$ of TIA patients, ${ }^{56,124,125}$ and DWI positivity in TIA patients is associated with a higher risk of recurrent ischemic events. ${ }^{126}$ The distribution of the DWI lesions can help with the determination of the stroke etiology (scattered emboli in multiple territories indicative of proximal embolic source [eg, cardiac], watershed distribution of lesions suggestive of carotid disease, and so forth). ${ }^{127-129}$

MR-based perfusion imaging, either with dynamic susceptibility contrast or ASL, may additionally identify a vascular etiology in TIA patients. ${ }^{130,131}$

CTA or MRA of the intracranial and cervical arteries and duplex sonography (DUS) for the cervical arteries are used to identify stenosis and/or occlusion (level Ib) ${ }^{123}$ and determine appropriate secondary prevention, such as extracranial carotid revascularization, for these patients. An appropriate evaluation for cardiac sources of TIA/stroke (eg, echocardiography) should also be performed.

Summary. When revascularization therapy is not indicated or available, multimodal neuroimaging of the brain and cerebrovasculature with MR imaging should be performed to confirm the diagnosis of stroke, identify the underlying etiology, and assess immediate complications and risk of future stroke. ${ }^{123}$

1) Multimodal CT, including NCCT and CTA and possibly PCT, should be reserved for patients who have contraindications to MR imaging, or if MR imaging is not available. ${ }^{123}$

\section{Rationale and Imaging Evidence for Acute Ischemic Stroke Patients with Wake-Up Stroke or More Generally with Unknown Time of Onset}

Acute stroke patients presenting without a definite time of symptom onset, such as wake-up stroke, may or may not proceed to thrombolytic treatment. If no acute reperfusion therapy is considered, NCCT is recommended to assess for intracranial hemorrhage. Further imaging evaluation is consistent with recommendations discussed in the previous sections.
However, if acute reperfusion therapy is considered, typically as part of a clinical trial, multimodal MR imaging (by using the DWI-PWI mismatch or the DWI-FLAIR mismatch) or multimodal CT (NCCT, CTA, and PCT) is required to assess the "tissue clock," as the time clock concept does not apply. ${ }^{132-134}$

Summary. In acute stroke patients without a definite time of symptom onset, imaging recommendations depend on whether acute reperfusion therapy may be performed.

1) If no acute reperfusion therapy will be performed, imaging recommendations are consistent with those in the previous sections.

2) If acute reperfusion therapy is considered, multimodal MR imaging or CT with perfusion imaging is recommended to evaluate viable tissue, as the time clock is not applicable. However, there is no firm evidence supporting imaging selection for treatment in this patient population.

\section{Rationale and Imaging Evidence for Patients Suspected of Posterior Fossa Stroke}

Acute stroke imaging in patients presenting with posterior fossa infarctions is quite similar to hemispheric ischemic stroke. A few aspects specific to the posterior fossa include the following:

- NCCT is relatively insensitive in detecting acute and small cortical or subcortical infarctions, especially in the posterior fossa. PCT has very limited indications for the posterior fossa as beam-hardening artifacts from the temporal bones limit the image quality. Additionally, the spatial resolution of PCT is challenged by the small size of ischemic lesions in the posterior fossa. Of further consideration, PCT imaging of the posterior fossa may involve inclusion of the ocular lenses in the cine imaging acquisition, which is associated with a non-negligible deterministic risk of cataract formation.

- MR imaging with DWI is the optimal imaging technique to assess for ischemic lesions in the posterior fossa (level Ia). ${ }^{130}$ It can assess the degree of brain stem infarction before intra-arterial treatment. However, due to the dismal prognosis of basilar occlusion, a higher risk is often tolerated to achieve recanalization at any time point.

- CTA, MRA, and DSA are the preferred imaging techniques to assess for basilar artery thrombosis (level Ia).

Summary. In acute stroke patients presenting with posterior fossa infarction, imaging recommendations are similar to hemispheric acute ischemic stroke.

1) MR imaging with DWI is the optimal imaging technique to assess the presence and extent of ischemia in the posterior fossa.

2) CTA and DSA are the preferred imaging techniques to assess for basilar artery thrombosis. MRA is an acceptable alternative for patients already undergoing an MR imaging examination.

\section{Rationale and Evidence Supporting Imaging of the Cervical Arteries in Acute Stroke and TIA Patients}

Imaging of the cervical arteries (in addition to imaging of the intracranial arteries) should be performed routinely as part of the imaging evaluation of patients with acute ischemic stroke but should not delay IV tPA administration in the first 4.5 hours. ${ }^{4,64}$ Similarly, noninvasive imaging of the cervical arteries should be a 
routine component of the imaging work-up of patients with TIAs. ${ }^{4,64}$ The primary goal of imaging the cervical arteries is to help identify the mechanism of the stroke and thus potentially to prevent a recurrence. ${ }^{4,64,135}$ Several imaging techniques are available to assess the cervical arteries including DUS, CTA, MRA, and DSA. ${ }^{136-138}$ Each technique has its own advantages and limitations in specific clinical situations, but overall, these noninvasive techniques show general agreement with DSA in approximately $90 \%$ of cases (level Ib). ${ }^{139-141}$ DSA is considered the reference standard imaging technique to assess the degree of stenosis and determine patient eligibility for carotid endarterectomy/angioplasty/stent placement. The concordant results of 2 noninvasive techniques (DUS, CTA, and/or MRA) can be used to determine treatment eligibility, avoiding catheterization risks. ${ }^{142,143}$ A 99\% stenosis (the so-called string sign) is most accurately detected by DSA, followed closely by CTA and contrast-enhanced MRA. ${ }^{144}$

Summary. In acute stroke patients, vascular imaging should be performed to evaluate the mechanism of stroke and assess risk of future stroke. ${ }^{1}$

1) Overall, vascular imaging with DUS, CTA, MRA, or DSA has good agreement.

2) Concordant results from at least 2 noninvasive imaging techniques can be used to determine treatment eligibility for revascularization procedures.

\section{ACKNOWLEDGMENTS}

We would like to thank Judy Burleson, MHSA, Director, Metrics, American College of Radiology and Christine Waldrip, RN, MHA, Program Manager, American College of Radiology Appropriateness Criteria, for the support they provided in the preparation of this manuscript.

\section{REFERENCES}

1. Rowley HA. Extending the time window for thrombolysis: evidence from acute stroke trials. Neuroimaging Clin N Am 2005;15: 575-87, $\mathrm{x}$

2. Sims J, Schwamm LH. The evolving role of acute stroke imaging in intravenous thrombolytic therapy: patient selection and outcomes assessment. Neuroimaging Clin N Am 2005;15:421-40, xii

3. Schellinger PD. The evolving role of advanced $M R$ imaging as a management tool for adult ischemic stroke: a Western-European perspective. Neuroimaging Clin N Am 2005;15:245-58, ix

3a. Oxford University. Oxford Centre for Evidence-based Medicine Levels of Evidence (March 2009) http://www.cebm.net/?O=1025 Accessed June 26, 2013.

4. DeLaPaz RL, Wippold FJ 2nd, Cornrlius RS, et al. ACR appropriateness criteria ${ }^{\circledR}$ on cerebrovascular disease. J Am Coll Radiol 2011;8:532-38

5. von Kummer R, Bourquain H, Bastianello S, et al. Early prediction of irreversible brain damage after ischemic stroke at CT. Radiology 2001;219:95-100

6. Paxton R, Ambrose J. The EMI scanner: a brief review of the first 650 patients. Br J Radiol 1974; 47:530-65

7. Jacobs L, Kinkel WR, Heffner RR Jr. Autopsy correlations of computerized tomography: experience with 6,000 CT scans. Neurology 1976;26:1111-18

8. Fiebach JB, Schellinger PD, Gass A, et al. Stroke magnetic resonance imaging is accurate in hyperacute intracerebral hemorrhage: a multicenter study on the validity of stroke imaging. Stroke 2004;35:502-06

9. Kidwell CS, Chalela JA, Saver JL, et al. Comparison of MRI and CT for detection of acute intracerebral hemorrhage. JAMA 2004;292:1823-30

10. Kakuda W, Thijs VN, Lansberg MG, et al. Clinical importance of microbleeds in patients receiving IV thrombolysis. Neurology 2005;65:1175-78

11. Lee SH, Kang BS, Kim N, et al. Does microbleed predict haemorrhagic transformation after acute atherothrombotic or cardioembolic stroke? J Neurol Neurosurg Psychiatry 2008;79:913-16

12. Boulanger JM, Coutts SB, Eliasziw M, et al. Cerebral microhemorrhages predict new disabling or fatal strokes in patients with acute ischemic stroke or transient ischemic attack. Stroke 2006;37:911-14

13. Fiehler J, Albers GW, Boulanger JM, et al. Bleeding Risk Analysis in Stroke Imaging before Thrombolysis (BRASIL): pooled analysis of $\mathrm{T} 2^{*}$-weighted magnetic resonance imaging data from 570 patients. Stroke 2007;38:2738-44

14. Kidwell CS, Wintermark M. Imaging of intracranial haemorrhage. Lancet Neurol 2008;7:256-67

15. Huisman TA. Intracranial hemorrhage: ultrasound, CT and MRI findings. Eur Radiol 2005;15:434-40

16. Hoggard N, Wilkinson ID, Paley MN, et al. Imaging of haemorrhagic stroke. Clin Radiol 2002;57:957-68

17. Kim J, Smith A, Hemphill JC, et al. Contrast extravasation on CT predicts mortality in primary intracerebral hemorrhage. AJNR Am J Neuroradiol 2008;29:520-25

18. Demchuk AM, Dowlatshahi D, Rodriguez-Luna D, et al. Prediction of haematoma growth and outcome in patients with intracerebral haemorrhage using the CT-angiography spot sign (PREDICT): a prospective observational study. Lancet Neurol 2012;11:307-14

19. Brouwers HB, Falcone GJ, McNamara KA, et al. CTA spot sign predicts hematoma expansion in patients with delayed presentation after intracerebral hemorrhage. Neurocrit Care 2012;17:421-28

20. Koculym A, Huynh TJ, Jakubovic R, et al. CT perfusion spot sign improves sensitivity for prediction of outcome compared with CTA and postcontrast CT. AJNR Am J Neuroradiol 2013;34:965-70

21. European Stroke Organisation (ESO) Executive Committee; ESO Writing Committee. Guidelines for management of ischaemic stroke and transient ischaemic attack 2008. Cerebrovasc Dis 2008;25:457-507

22. Saver JL. Time is brain-quantified. Stroke 2006;37:263-66

23. Wahlgren N, Ahmed N, Eriksson N, et al. Multivariable analysis of outcome predictors and adjustment of main outcome results to baseline data profile in randomized controlled trials: Safe Implementation of Thrombolysis in Stroke-Monitoring Study (SITSMOST). Stroke 2008;39:3316-22

24. Tissue plasminogen activator for acute ischemic stroke: the $\mathrm{Na}$ tional Institute of Neurological Disorders and Stroke rt-PA Stroke Study Group. N Engl J Med 1995;333:1581-87

25. Adams HP Jr, Brott TG, Furlan AJ, et al. Guidelines for thrombolytic therapy for acute stroke: a supplement to the guidelines for the management of patients with acute ischemic stroke-a statement for healthcare professionals from a Special Writing Group of the Stroke Council, American Heart Association. Circulation 1996;94:1167-74

26. Hacke W, Kaste M, Fieschi C, et al. Intravenous thrombolysis with recombinant tissue plasminogen activator for acute hemispheric stroke: the European Cooperative Acute Stroke Study (ECASS). JAMA 1995;274:1017-25

27. Hacke W, Kaste M, Bluhmki E, et al. Thrombolysis with alteplase 3 to 4.5 hours after acute ischemic stroke. $N$ Engl $\mathrm{J} \mathrm{Med}$ 2008;359:1317-29

28. Bluhmki E, Chamorro A, Davalos A, et al. Stroke treatment with alteplase given $3.0-4.5 \mathrm{~h}$ after onset of acute ischaemic stroke (ECASS III): additional outcomes and subgroup analysis of a randomised controlled trial. Lancet Neurol 2009;8:1095-102

29. Lees KR, Bluhmki E, von Kummer R, et al. Time to treatment with intravenous alteplase and outcome in stroke: an updated pooled 
analysis of ECASS, ATLANTIS, NINDS, and EPITHET trials. Lancet 2010;375:1695-703

30. von Kummer R, Meyding-Lamade U, Forsting M, et al. Sensitivity and prognostic value of early CT in occlusion of the middle cerebral artery trunk. AJNR Am J Neuroradiol 1994;15:9-15, discussion $16-18$

31. Larrue V, von Kummer RR, Muller A, et al. Risk factors for severe hemorrhagic transformation in ischemic stroke patients treated with recombinant tissue plasminogen activator: a secondary analysis of the European-Australasian acute stroke study (ECASS II). Stroke 2001;32:438-41

32. Lee KH, Lee SJ, Cho SJ, et al. Usefulness of triphasic perfusion computed tomography for intravenous thrombolysis with tissuetype plasminogen activator in acute ischemic stroke. Arch Neurol 2000;57:1000-08

33. Lev MH, Segal AZ, Farkas J, et al. Utility of perfusion-weighted CT imaging in acute middle cerebral artery stroke treated with intraarterial thrombolysis: prediction of final infarct volume and clinical outcome. Stroke 2001;32:2021-28

34. Wintermark M, Reichhart M, Thiran JP, et al. Prognostic accuracy of cerebral blood flow measurement by perfusion computed tomography, at the time of emergency room admission, in acute stroke patients. Ann Neurol 2002;51:417-32

35. Koroshetz WJ, Lev MH. Contrast computed tomography scan in acute stroke: "You can't always get what you want but you get what you need." Ann Neurol 2002;51:415-16

36. Patel SC, Levine SR, Tilley BC, et al. Lack of clinical significance of early ischemic changes on computed tomography in acute stroke. JAMA 2001;286:2830-38

37. von Kummer R, Nolte PN, Schnittger H, et al. Detectability of cerebral hemisphere ischaemic infarcts by CT within $6 \mathrm{~h}$ of stroke. Neuroradiology 1996;38:31-33

38. Schellinger PD, Fiebach JB, Hacke W. Imaging-based decision making in thrombolytic therapy for ischemic stroke: present status. Stroke 2003;34:575-83

39. Schriger DL, Kalafut M, Starkman S, et al. Cranial computed tomography interpretation in acute stroke: physician accuracy in determining eligibility for thrombolytic therapy. JAMA 1998;279:1293-97

40. Grotta JC, Chiu D, Lu M, et al. Agreement and variability in the interpretation of early CT changes in stroke patients qualifying for intravenous rtPA therapy. Stroke 1999;30:1528-33

41. Kalafut MA, Schriger DL, Saver JL, et al. Detection of early CT signs of $>1 / 3$ middle cerebral artery infarctions: interrater reliability and sensitivity of CT interpretation by physicians involved in acute stroke care. Stroke 2000;31:1667-71

42. Barber PA, Demchuk AM, Zhang J, et al. Validity and reliability of a quantitative computed tomography score in predicting outcome of hyperacute stroke before thrombolytic therapy; aspects study group-Alberta Stroke Programme Early CT Score. Lancet 2000;355:1670-74

43. Pexman JH, Barber PA, Hill MD, et al. Use of the Alberta Stroke Program Early CT Score (ASPECTS) for assessing CT scans in patients with acute stroke. AJNR Am J Neuroradiol 2001;22:1534-42

44. Demchuk AM, Coutts SB. Alberta Stroke Program Early CT Score in Acute Stroke Triage. Neuroimaging Clin N Am 2005;15:409-19, xii

45. Schramm P, Schellinger PD, Fiebach JB, et al. Comparison of CT and $\mathrm{CT}$ angiography source images with diffusion-weighted imaging in patients with acute stroke within 6 hours after onset. Stroke 2002;33:2426-32

46. Schramm P, Schellinger PD, Klotz E, et al. Comparison of perfusion computed tomography and computed tomography angiography source images with perfusion-weighted imaging and diffusionweighted imaging in patients with acute stroke of less than 6 hours' duration. Stroke 2004;35:1652-58

47. Sharma M, Fox AJ, Symons S, et al. CT angiographic source images: flow- or volume-weighted? AJNR Am J Neuroradiol 2011;32:359-64

48. Chalela JA, Kidwell CS, Nentwich LM, et al. Magnetic resonance imaging and computed tomography in emergency assessment of patients with suspected acute stroke: a prospective comparison. Lancet 2007;369:293-98

49. Fiebach JB, Schellinger PD, Jansen $\mathrm{O}$, et al. CT and diffusionweighted MR imaging in randomized order: diffusion-weighted imaging results in higher accuracy and lower interrater variability in the diagnosis of hyperacute ischemic stroke. Stroke 2002;33:2206-10

50. González RG, Schaefer PW, Buonanno FS, et al. Diffusion-weighted MR imaging: diagnostic accuracy in patients imaged within 6 hours of stroke symptom onset. Radiology 1999;210:155-62

51. Kucharczyk J, Mintorovitch J, Asgari HS, et al. Diffusion/perfusion MR imaging of acute cerebral ischemia. Magn Reson Med 1991;19:311-15

52. Warach S, Gaa J, Siewert B, et al. Acute human stroke studied by whole brain echo planar diffusion-weighted magnetic resonance imaging. Ann Neurol 1995;37:231-41

53. Brazzelli M, Sandercock PA, Chappell FM, et al. Magnetic resonance imaging versus computed tomography for detection of acute vascular lesions in patients presenting with stroke symptoms. Cochrane Database Syst Rev 2009:CD007424

54. Lövblad KO, Laubach HJ, Baird AE, et al. Clinical experience with diffusion-weighted MR in patients with acute stroke. AJNR Am J Neuroradiol 1998;19:1061-66

55. Marks MP, de Crespigny A, Lentz D, et al. Acute and chronic stroke: navigated spin-echo diffusion-weighted MR imaging. Radiology 1996;199:403-08

56. Kidwell CS, Alger JR, Di Salle F, et al. Diffusion MRI in patients with transient ischemic attacks. Stroke 1999;30:1174-80

57. Ay H, Buonanno FS, Rordorf G, et al. Normal diffusion-weighted MRI during stroke-like deficits. Neurology 1999;52:1784-92

58. Barber PA, Darby DG, Desmond PM, et al. Identification of major ischemic change: diffusion-weighted imaging versus computed tomography. Stroke 1999;30:2059-65

59. Lee LJ, Kidwell CS, Alger J, et al. Impact on stroke subtype diagnosis of early diffusion-weighted magnetic resonance imaging and magnetic resonance angiography. Stroke 2000;31:1081-89

60. Warach S, Pettigrew LC, Dashe JF, et al. Effect of citicoline on ischemic lesions as measured by diffusion-weighted magnetic resonance imaging: Citicoline 010 Investigators. Ann Neurol 2000;48:713-22

61. Tong DC, Yenari MA, Albers GW, et al. Correlation of perfusionand diffusion-weighted MRI with NIHSS score in acute $(<6.5$ hour) ischemic stroke. Neurology 1998;50:864-70

62. Lövblad KO, Baird AE, Schlaug G, et al. Ischemic lesion volumes in acute stroke by diffusion-weighted magnetic resonance imaging correlate with clinical outcome. Ann Neurol 1997;42:164-70

63. Latchaw RE, Alberts MJ, Lev MH, et al. Recommendations for imaging of acute ischemic stroke: a scientific statement from the American Heart Association. Stroke 2009;40:3646-78

64. Jauch E, Saver J, Adams HP, et al. Guidelines for the early management of patients with acute ischemic stroke: a guideline for healthcare professionals from the American Heart Association/American Stroke Association. Stroke 2013;44:870-947

65. Kidwell CS, Jahan R, Gornbein J, et al. A trial of imaging selection and endovascular treatment for ischemic stroke. $N$ Engl J Med 2013;368:914-23

66. Broderick JP, Palesch YY, Demchuk AM, et al. Endovascular therapy after intravenous T-PA versus T-PA alone for stroke. $N$ Engl J Med 2013;368:893-903

67. Ciccone A, Valvassori L, Nichelatti M, et al, for the SYNTHESIS Expansion Investigators. Endovascular treatment for acute ischemic stroke. N Engl J Med 2013;368:904-13

68. Rha JH, Saver JL. The impact of recanalization on ischemic stroke outcome: a meta-analysis. Stroke 2007;38:967-73 
69. Caplan LR. Treatment of acute stroke: still struggling. JAMA 2004;292:1883-85

70. Caplan LR. Stroke thrombolysis: slow progress. Circulation 2006;114:187-90

71. Saqqur M, Uchino K, Demchuk AM, et al. Site of arterial occlusion identified by transcranial Doppler predicts the response to intravenous thrombolysis for stroke. Stroke 2007;38:948-54

72. Sims JR, Rordorf G, Smith EE, et al. Arterial occlusion revealed by CT angiography predicts NIH stroke score and acute outcomes after IV TPA treatment. AJNR Am J Neuroradiol 2005;26:246-51

73. Ogawa A, Mori E, Minematsu K, et al. Randomized trial of intraarterial infusion of urokinase within 6 hours of middle cerebral artery stroke: the middle cerebral artery embolism local fibrinolytic intervention trial (MELT) Japan. Stroke 2007;38:2633-39

74. Saver JL. Intra-arterial fibrinolysis for acute ischemic stroke: the message of MELT. Stroke 2007;38:2627-28

75. Marder VJ, Chute DJ, Starkman S, et al. Analysis of thrombi retrieved from cerebral arteries of patients with acute ischemic stroke. Stroke 2006;37:2086-93

76. Liebeskind DS, Sanossian N, Yong WH, et al. CT and MRI early vessel signs reflect clot composition in acute stroke. Stroke 2011;42:1237-43

77. Moftakhar P, English JD, Cooke DL, et al. Density of thrombus on admission CT predicts revascularization efficacy in large vessel occlusion acute ischemic stroke. Stroke 2013;44:243-45

78. Albers GW, Thijs VN, Wechsler L, et al. Magnetic resonance imaging profiles predict clinical response to early reperfusion: the diffusion and perfusion imaging evaluation for understanding stroke evolution (DEFUSE) study. Ann Neurol 2006;60:508-17

79. Lansberg MG, Straka M, Kemp S, et al. MRI profile and response to endovascular reperfusion after stroke (DEFUSE 2): a prospective cohort study. Lancet Neurol 2012;11:860-67

80. Schellinger PD, Bryan RN, Caplan LR, et al. Evidence-based guideline: the role of diffusion and perfusion MRI for the diagnosis of acute ischemic stroke-report of the Therapeutics and Technology Assessment Subcommittee of the American Academy of Neurology. Neurology 2010;75:177-85

81. Breuer L, Schellinger PD, Huttner HB, et al. Feasibility and safety of magnetic resonance imaging-based thrombolysis in patients with stroke on awakening: initial single-centre experience. Int J Stroke 2010;5:68-73

82. Köhrmann M, Schellinger PD. Stroke-MRI: extending the timewindow-recent trials and clinical practice. Int $J$ Stroke 2007;2:53-54

83. Hirai T, Korogi Y, Ono K, et al. Prospective evaluation of suspected stenoocclusive disease of the intracranial artery: combined MR angiography and CT angiography compared with digital subtraction angiography. AJNR Am J Neuroradiol 2002;23:93-101

84. Katz DA, Marks MP, Napel SA, et al. Circle of Willis: evaluation with spiral CT angiography, MR angiography, and conventional angiography. Radiology 1995;195:445-49

85. Knauth M, von Kummer R, Jansen O, et al. Potential of CT angiography in acute ischemic stroke. AJNR Am J Neuroradiol 1997;18:1001-10

86. Shrier DA, Tanaka H, Numaguchi $\mathrm{Y}$, et al. CT angiography in the evaluation of acute stroke. AJNR Am J Neuroradiol 1997;18:1011-20

87. Wildermuth S, Knauth M, Brandt T, et al. Role of CT angiography in patient selection for thrombolytic therapy in acute hemispheric stroke. Stroke 1998;29:935-38

88. Verro P, Tanenbaum LN, Borden NM, et al. CT angiography in acute ischemic stroke: preliminary results. Stroke 2002;33:276-78

89. Graf J, Skutta B, Kuhn FP, et al. Computed tomographic angiography findings in 103 patients following vascular events in the posterior circulation: potential and clinical relevance. $J$ Neurol 2000;247:760-66

90. Nguyen-Huynh MN, Wintermark M, English J, et al. How accurate is $\mathrm{CT}$ angiography in evaluating intracranial atherosclerotic disease? Stroke 2008;39:1184-88

91. Bash S, Villablanca JP, Jahan R, et al. Intracranial vascular stenosis and occlusive disease: evaluation with CT angiography, MR angiography, and digital subtraction angiography. AJNR Am J Neuroradiol 2005;26:1012-21

92. Pulli B, Schaefer PW, Hakimelahi R, et al. Acute ischemic stroke: infarct core estimation on CT angiography source images depends on CT angiography protocol. Radiology 2012;262:593-604

93. Hacke W, Furlan AJ, Al-Rawi Y, et al. Intravenous desmoteplase in patients with acute ischaemic stroke selected by MRI perfusiondiffusion weighted imaging or perfusion CT (DIAS-2): a prospective, randomised, double-blind, placebo-controlled study. Lancet Neurol 2009;8:141-50

94. Davis SM, Donnan GA, Parsons MW, et al. Effects of alteplase beyond $3 \mathrm{~h}$ after stroke in the Echoplanar Imaging Thrombolytic Evaluation Trial (EPITHET): a placebo-controlled randomised trial. Lancet Neurol 2008;7:299-309

95. Butcher KS, Parsons M, MacGregor L, et al. Refining the perfusiondiffusion mismatch hypothesis. Stroke 2005;36:1153-59

96. Parsons MW, Barber PA, Chalk J, et al. Diffusion- and perfusionweighted MRI response to thrombolysis in stroke. Ann Neurol 2002;51:28-37

97. Sorensen AG, Copen WA, Ostergaard L, et al. Hyperacute stroke: simultaneous measurement of relative cerebral blood volume, relative cerebral blood flow, and mean tissue transit time. Radiology 1999;210:519-27

98. Perkins CJ, Kahya E, Roque CT, et al. Fluid-attenuated inversion recovery and diffusion- and perfusion-weighted MRI abnormalities in 117 consecutive patients with stroke symptoms. Stroke 2001;32:2774-81

99. Furlan AJ, Eyding D, Albers GW, et al. Dose escalation of Desmoteplase for Acute Ischemic Stroke (DEDAS): evidence of safety and efficacy 3 to 9 hours after stroke onset. Stroke 2006;37:1227-31

100. Hacke W, Albers G, Al-Rawi Y, et al. The Desmoteplase in Acute Ischemic Stroke trial (DIAS): a phase II MRI-based 9-hour window acute stroke thrombolysis trial with intravenous desmoteplase. Stroke 2005;36:66-73

101. Lansberg MG, Lee J, Christensen S, et al. Rapid automated patient selection for reperfusion therapy: a pooled analysis of the Echoplanar Imaging Thrombolytic Evaluation Trial (EPITHET) and the Diffusion and Perfusion Imaging Evaluation for Understanding Stroke Evolution (DEFUSE) study. Stroke 2011;42:1608-14

102. Mlynash M, Lansberg MG, De Silva DA, et al. Refining the definition of the malignant profile: insights from the DEFUSEEPITHET pooled data set. Stroke 2011;42:1270-75

103. Olivot JM, Mlynash M, Thijs VN, et al. Optimal Tmax threshold for predicting penumbral tissue in acute stroke. Stroke 2009;40:469-75

104. Wintermark M, Flanders AE, Velthuis B, et al. Perfusion-CT assessment of infarct core and penumbra: receiver operating characteristic curve analysis in 130 patients suspected of acute hemispheric stroke. Stroke 2006;37:979-85

105. Mayer TE, Hamann GF, Baranczyk J, et al. Dynamic CT perfusion imaging of acute stroke. AJNR Am J Neuroradiol 2000;21:1441-49

106. Bivard A, McElduff P, Spratt N, et al. Defining the extent of irreversible brain ischemia using perfusion computed tomography. Cerebrovasc Dis 2011;31:238-45

107. Arakawa S, Wright PM, Koga M, et al. Ischemic thresholds for gray and white matter: a diffusion and perfusion magnetic resonance study. Stroke 2006;37:1211-16

108. Sobesky J, Zaro Weber O, Lehnhardt FG, et al. Does the mismatch match the penumbra? Magnetic resonance imaging and positron emission tomography in early ischemic stroke. Stroke 2005;36:980-85

109. Bivard A, Levi C, Spratt N, et al. Perfusion CT in acute stroke: a comprehensive analysis of infarct and penumbra. Radiology 2013;267:543-50 
110. Bivard A, Spratt N, Levi C, et al. Perfusion computer tomography: imaging and clinical validation in acute ischaemic stroke. Brain 2011;134:3408-16

111. Kamalian S, Kamalian S, Maas MB, et al. CT cerebral blood flow maps optimally correlate with admission diffusion-weighted imaging in acute stroke but thresholds vary by postprocessing platform. Stroke 2011;42:1923-28

112. Payabvash S, Souza LC, Wang Y, et al. Regional ischemic vulnerability of the brain to hypoperfusion: the need for location specific computed tomography perfusion thresholds in acute stroke patients. Stroke 2011;42:1255-60

113. Kloska SP, Nabavi DG, Gaus C, et al. Acute stroke assessment with CT: do we need multimodal evaluation? Radiology 2004;233:79-86

114. Wintermark M, Fischbein NJ, Smith WS, et al. Accuracy of dynamic perfusion CT with deconvolution in detecting acute hemispheric stroke. AJNR Am J Neuroradiol 2005;26:104-12

115. Scharf J, Brockmann MA, Daffertshofer M, et al. Improvement of sensitivity and interrater reliability to detect acute stroke by dynamic perfusion computed tomography and computed tomography angiography. J Comput Assist Tomogr 2006;30:105-10

116. Keedy A, Soares B, Wintermark M. A pictorial essay of brain perfusion-CT: not every abnormality is a stroke! J Neuroimaging 2012;22:e20-33

117. Tan JC, Dillon WP, Liu S, et al. Systematic comparison of perfusion-CT and CT-angiography in acute stroke patients. Ann Neurol 2007;61:533-43

118. Ryoo JW, Na DG, Kim SS, et al. Malignant middle cerebral artery infarction in hyperacute ischemic stroke: evaluation with multiphasic perfusion computed tomography maps. J Comput Assist Tomogr 2004;28:55-62

119. Hom J, Dankbaar JW, Soares BP, et al. Blood-brain barrier permeability assessed by perfusion CT predicts symptomatic hemorrhagic transformation and malignant edema in acute ischemic stroke. AJNR Am J Neuroradiol 2011;32:41-48

120. Bang OY, Saver JL, Kim SJ, et al. Collateral flow predicts response to endovascular therapy for acute ischemic stroke. Stroke 2011;42:693-99

121. Kim SJ, Seok JM, Bang OY, et al. MR mismatch profiles in patients with intracranial atherosclerotic stroke: a comprehensive approach comparing stroke subtypes. J Cereb Blood Flow Metab 2009;29:1138-45

122. del Zoppo GJ, Sharp FR, Heiss WD, et al. Heterogeneity in the penumbra. J Cereb Blood Flow Metab 2011;31:1836-51

123. Easton JD, Saver JL, Albers GW, et al. Definition and evaluation of transient ischemic attack: a scientific statement for healthcare professionals from the American Heart Association/American Stroke Association Stroke Council; Council on Cardiovascular Surgery and Anesthesia; Council on Cardiovascular Radiology and Intervention; Council on Cardiovascular Nursing; and the Interdisciplinary Council on Peripheral Vascular Disease-The American Academy of Neurology affirms the value of this statement as an educational tool for neurologists. Stroke 2009;40:2276-93

124. Coutts SB, Hill MD, Simon JE, et al. Silent ischemia in minor stroke and TIA patients identified on MR imaging. Neurology 2005;65:513-17

125. Restrepo L, Jacobs MA, Barker PB, et al. Assessment of transient ischemic attack with diffusion- and perfusion-weighted imaging. AJNR Am J Neuroradiol 2004;25:1645-52

126. Cucchiara BL, Messe SR, Taylor RA, et al. Is the ABCD score useful for risk stratification of patients with acute transient ischemic attack? Stroke 2006;37:1710-14

127. Kang DW, Chalela JA, Ezzeddine MA, et al. Association of ischemic lesion patterns on early diffusion-weighted imaging with TOAST stroke subtypes. Arch Neurol 2003;60:1730-34

128. Wessels T, Wessels C, Ellsiepen A, et al. Contribution of diffusionweighted imaging in determination of stroke etiology. AJNR Am J Neuroradiol 2006;27:35-39

129. Rovira A, Grive E, Rovira A, et al. Distribution territories and causative mechanisms of ischemic stroke. Eur Radiol 2005;15:416-26

130. Mlynash M, Olivot JM, Tong DC, et al. Yield of combined perfusion and diffusion MR imaging in hemispheric TIA. Neurology 2009;72:1127-33

131. Zaharchuk G, Olivot JM, Fischbein NJ, et al. Arterial spin labeling imaging findings in transient ischemic attack patients: comparison with diffusion- and bolus perfusion-weighted imaging. Cerebrovasc Dis 2012;34:221-28

132. Thomalla G, Ebinger M, Fiehler J, et al. EU-funded treatment study: WAKE-UP - a randomized, placebo-controlled MRI-based trial of thrombolysis in wake-up stroke [in German]. Nervenarzt 2012;83:1241-51

133. Thomalla G, Cheng B, Ebinger M, et al. DWI-FLAIR mismatch for the identification of patients with acute ischaemic stroke within $4.5 \mathrm{~h}$ of symptom onset (PRE-FLAIR): a multicentre observational study. Lancet Neurol 2011;10:978-86

134. Kang DW, Kwon JY, Kwon SU, et al. Wake-up or unclear-onset strokes: are they waking up to the world of thrombolysis therapy? Int J Stroke 2012;7:311-20

135. Adams RJ, Albers G, Alberts MJ, et al. Update to the AHA/ASA recommendations for the prevention of stroke in patients with stroke and transient ischemic attack. Stroke 2008;39:1647-52

136. Buskens E, Nederkoorn PJ, Buijs-Van Der Woude T, et al. Imaging of carotid arteries in symptomatic patients: cost-effectiveness of diagnostic strategies. Radiology 2004;233:101-12

137. Lovett JK, Dennis MS, Sandercock PA, et al. Very early risk of stroke after a first transient ischemic attack. Stroke 2003;34:e138-140

138. Rothwell PM, Giles MF, Flossmann E, et al. A simple score (ABCD) to identify individuals at high early risk of stroke after transient ischaemic attack. Lancet 2005;366:29-36

139. Blakeley DD, Oddone EZ, Hasselblad V, et al. Noninvasive carotid artery testing: a meta-analytic review. Ann Intern Med 1995; 122:360-67

140. Long A, Lepoutre A, Corbillon E, et al. Critical review of non- or minimally invasive methods (duplex ultrasonography, MR- and CT-angiography) for evaluating stenosis of the proximal internal carotid artery. Eur J Vasc Endovasc Surg 2002;24:43-52

141. Randoux B, Marro B, Koskas F, et al. Carotid artery stenosis: prospective comparison of $\mathrm{CT}$, three-dimensional gadoliniumenhanced MR, and conventional angiography. Radiology 2001;220:179-85

142. Johnston DC, Goldstein LB. Clinical carotid endarterectomy decision making: noninvasive vascular imaging versus angiography. Neurology 2001;56:1009-15

143. Nederkoorn PJ, Mali WP, Eikelboom BC, et al. Preoperative diagnosis of carotid artery stenosis: accuracy of noninvasive testing. Stroke 2002;33:2003-08

144. Lev MH, Romero JM, Goodman DN, et al. Total occlusion versus hairline residual lumen of the internal carotid arteries: accuracy of single section helical CT angiography. AJNR Am J Neuroradiol 2003;24:1123-29 